TITLE:

\title{
Mott transition and heavy-fermion state in the pyrochlore Hubbard model
}

\author{
$\operatorname{AUTHOR}(\mathrm{S}):$
}

Fujimoto, $S$

\section{CITATION:}

Fujimoto, S. Mott transition and heavy-fermion state in the pyrochlore Hubbard model. PHYSICAL REVIEW B 2001, 64(8): 085102.

ISSUE DATE:

2001-08-15

URL:

http://hdl.handle.net/2433/50018

RIGHT:

Copyright 2001 American Physical Society 
PHYSICAL REVIEW B, VOLUME 64, 085102

\title{
Mott transition and heavy-fermion state in the pyrochlore Hubbard model
}

\author{
Satoshi Fujimoto \\ Department of Physics, Kyoto University, Kyoto 606-8502, Japan \\ (Received 7 March 2001; revised manuscript received 26 April 2001; published 1 August 2001)
}

\begin{abstract}
We investigate the interplay between geometrical frustration and strong electron correlation based upon the pyrochlore Hubbard model. In the half-filling case, using the perturbative expansion in terms of electron correlation, we show that the self-energy shows a divergent behavior leading the system into the Mott insulating state, in which a quantum disordered spin liquid without magnetic long-range order is realized. In the hole-doped case, we obtain heavy-fermion-like Fermi-liquid state. We also calculate the neutron cross section, which is very consistent with recent neutron scattering experiments for itinerant pyrochlore systems.
\end{abstract}

DOI: 10.1103/PhysRevB.64.085102

PACS number(s): 71.27.+a, 71.30.+h, 75.10.-b, 75.40.Gb

\section{INTRODUCTION}

Recently, the role played by geometrical frustration in strongly correlated electron systems has attracted renewed interests. $^{1-6}$ The pyrochlore lattice, namely, corner-sharing tetrahedra (Fig. 1), is one of the typical system in which the geometrical frustration is crucial to determine its properties. Here we investigate effects of geometrical frustration on itinerant electron systems based upon the pyrochlore Hubbard model. One of the purpose of this paper is to investigate the Mott transition in the absence of magnetic long-range order. According to the study of the Heisenberg model on the pyrochlore lattice, it has been pointed out that the ground state of the insulating state is a quantum disordered spin liquid. $^{7-15}$ The critical character of the Mott transition without magnetic order is quite different from that accompanying the antiferromagnetic one. Moreover, the character of the quantum spin liquid has not yet been revealed sufficiently. It is expected that the study from electron systems will shed new light on this subject.

Another purpose of this paper is to reveal how the geo- metrical frustration affects strong electron correlation effects in the metallic state. Some recent experiments on the pyrochlore itinerant electron systems have reported that remarkable heavy-fermion-like behaviors manifest in these systems implying the crucial role of the geometrical frustration. ${ }^{1,2}$ Here we show that the heavy-fermion state is realized in the pyrochlore Hubbard model in the vicinity of the half-filling. We also show that the dynamical spin susceptibility obtained from our model away from the half-filling is consistent with recent neutron scattering experiments for itinerant pyrochlore systems. $^{16}$

The organization of this paper is as follows. The model and the basic method are given in Sec. II. In Sec. III, we discuss about the Mott transition and the realization of the quantum disordered spin liquid in the half-filling case. In Sec. IV, we consider the hole-doped case focusing on the heavy-fermion state and its magnetic properties.

\section{MODEL HAMILTONIAN AND METHOD}

The Hamiltonian of the model is given by
$H=-2 \sum_{k, \sigma} \psi_{k \sigma}^{\dagger}\left(\begin{array}{cccc}0 & \cos \left(k_{x}+k_{y}\right) & \cos \left(k_{y}-k_{z}\right) & \cos \left(k_{x}-k_{z}\right) \\ \cos \left(k_{x}+k_{y}\right) & 0 & \cos \left(k_{x}+k_{z}\right) & \cos \left(k_{y}+k_{z}\right) \\ \cos \left(k_{y}-k_{z}\right) & \cos \left(k_{x}+k_{z}\right) & 0 & \cos \left(k_{x}-k_{y}\right) \\ \cos \left(k_{x}-k_{z}\right) & \cos \left(k_{y}+k_{z}\right) & \cos \left(k_{x}-k_{y}\right) & 0\end{array}\right) \psi_{k \sigma}+U \sum_{i} \sum_{\nu=1}^{4} c_{i \nu \uparrow}^{\dagger} c_{i \nu \uparrow} c_{i \nu \downarrow}^{\dagger} c_{i \nu \downarrow}$

$$
\begin{aligned}
& \text { where } n_{ \pm}=\sqrt{s(x \pm z)^{2}+s(y \mp z)^{2}+s(x+y)^{2}} \text {, and } \\
& \begin{aligned}
\vec{s}_{2}(k)= & \left(s(x+z) s(x-z) s(y-z)-s(y+z)\left[s(y-z)^{2}+s(x\right.\right. \\
& \left.+y)^{2}\right],
\end{aligned} \\
& \quad \begin{array}{l}
s(x+z) s(y+z) s(y-z)-s(x-z)\left[s(x+z)^{2}+s(x+y)^{2}\right] \\
-s(x+y)[s(x+z) s(y+z)+s(x-z) s(y-z)],
\end{array} \\
& \left.\quad s(x+y) n_{+}^{2}\right) / n_{2},
\end{aligned}
$$

Here $c_{i \nu \sigma}\left(c_{i \nu \sigma}^{\dagger}\right)$ is an annihilation (creation) operator of electrons with spin $\sigma$ on the $\nu$ th site of the $i$ th tetrahedron. $\psi_{k \sigma}^{\dagger}=\left(c_{k, 1 \sigma}^{\dagger}, c_{k, 2 \sigma}^{\dagger}, c_{k, 3 \sigma}^{\dagger}, c_{k, 4 \sigma}^{\dagger}\right)$. The kinetic term is diagonalized as, $H_{\mathrm{kin}}=\sum_{k \sigma} \sum_{\nu=1}^{4} E_{k \nu} a_{k \nu \sigma}^{\dagger} a_{k \nu \sigma}$, where $E_{k 1}=E_{k 2}=1$, $E_{k 3}=-1+\sqrt{1+t_{k}}, \quad E_{k 4}=-1-\sqrt{1+t_{k}} \quad$ with $t_{k}$ $=\cos \left(2 k_{x}\right) \cos \left(2 k_{y}\right)+\cos \left(2 k_{y}\right) \cos \left(2 k_{z}\right)+\cos \left(2 k_{z}\right) \cos \left(2 k_{x}\right)$. The basis in the diagonalized space is obtained from the canonical transformation, $\psi_{k \sigma}^{\dagger}=\sum_{\nu=1}^{4} \vec{s}_{\nu}(k) a_{k \nu \sigma}^{\dagger}$. Using the abbreviation, $s(x \pm y) \equiv \sin \left(k_{x} \pm k_{y}\right)$ etc., we write down $\vec{s}_{1,2}(\mathrm{k})$,

$$
\vec{s}_{1}(k)=(s(x+z), s(y-z),-s(x+y), 0) / n_{+},
$$

for $k_{x}+k_{y} \neq 0$, where $n_{2}=n_{+}\left\{n_{+}^{2} n_{-}^{2}-[s(x+z) s(y+z)\right.$
$\left.+s(x-z) s(y-z)]^{2}\right\}^{1 / 2}$, and 


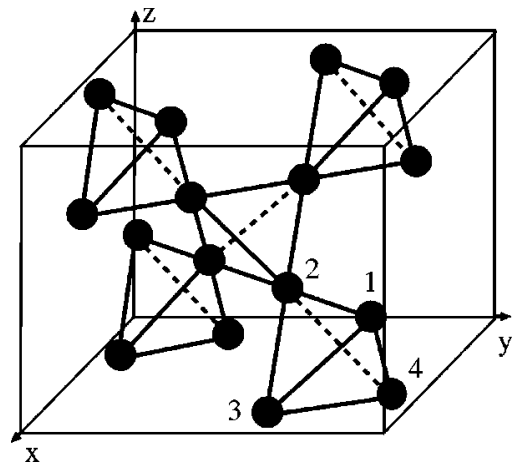

FIG. 1. Pyrochlore lattice.

$$
\begin{aligned}
\vec{s}_{2}(k)= & (-s(2 x),-s(2 x), 2 s(x-z), 2 s(x+z)) \\
& \times 1 / \sqrt{2 s(2 x)^{2}+4 s(x-z)^{2}+4 s(x+z)^{2}}
\end{aligned}
$$

for $k_{x}+k_{y}=0$. The expressions of $\vec{s}_{3}(k)$ and $\vec{s}_{4}(k)$ are very complicated. However, in the following, we need only $\vec{s}_{3}(k)$ for small $k$, which is given by

$$
\begin{gathered}
\vec{s}_{3}(k)=\left(-k_{x}-k_{y}+k_{z}, k_{x}+k_{y}+k_{z},\right. \\
\left.-k_{x}+k_{y}-k_{z}, k_{x}-k_{y}-k_{z}\right) / 2|k| .
\end{gathered}
$$

At the half-filling, in the absence of electron-electron interaction, $E_{k 3}$ and $E_{k 4}$ are filled completely, and the two degenerate flat bands are empty. $E_{k 3}$ touches with the two flat bands at the $\Gamma$ point of the Brillouin zone. This state is a gapless band insulator. We now consider the effect of electron interactions on this state. Since, in the case of the halffilling or in the vicinity of the half-filling, the band $E_{k 4}$ is sufficiently far from the Fermi level, it does not affect lowenergy properties and is negligible in the following argument. Then, the single-particle Green's functions of $a_{k \nu \sigma}$ electron are determined by the following equation:

$$
\sum_{\lambda=1}^{3}\left[\left(\varepsilon+\mu-E_{k \lambda}\right) \delta_{\mu \lambda}-\Sigma_{k}^{(\mu \lambda)}(\varepsilon)\right] G_{k}^{(\lambda \nu)}(\varepsilon)=\delta_{\mu \nu} .
$$

We put the chemical potential $\mu=1$. In general, the offdiagonal self-energy $\Sigma^{(\mu \nu)}(\mu \neq \nu)$ is not negligible. However, in the vicinity of the $\Gamma$ point, at which the most important scattering processes occur, the off-diagonal terms vanish because of the momentum dependence of $\vec{s}_{\nu}(k)$. Thus in the following argument, we neglect the off-diagonal self-energy.

\section{THE HALF-FILLING CASE}

\section{A. Self-energy}

To investigate the electronic state at the half-filling, we calculate the self-energy. Recently, Isoda and Mori obtained the diagonal self-energy of this model up to the second order in $U,{ }^{17}$ which is given by $\operatorname{Re} \Sigma(\varepsilon) \sim \sqrt{-\varepsilon}$ for $\varepsilon<0$. However, as will be shown here, the higher-order corrections to the self-energy give rise to more singular contributions and a drastic change of the electronic state, namely, the Mott transition.
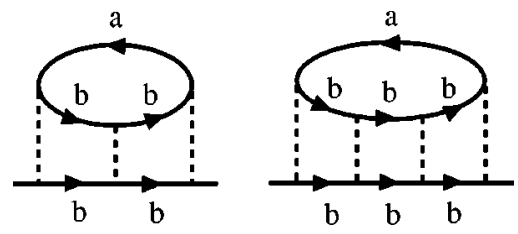

FIG. 2. The most singular diagrams of the self-energy up to the fourth order in $U . a$ denotes an $a_{3}$ electron. $b$ denotes an $a_{1}$ or $a_{2}$ electron. The dotted line denotes the interaction $U$.

At the half-filling, the $E_{k 3}$ band is completely filled, while the flat bands are empty. Hence, at zero temperature, the particle-particle channel between an $a_{3}$ electron and $a_{1}$ or $a_{2}$ electron vanishes. On the other hand, particle-hole channel gives nonvanishing contributions only for the pair of an $a_{3}$ electron and $a_{1}$ or $a_{2}$ electron. Then the most singular diagrams up to the forth order in $U$ are those shown in Fig. 2. Since the Fermi level is at the position between the two flat bands and $E_{k 3}$ band, we shift the chemical potential infinitesimally, $\mu \rightarrow 1-\delta$. After integrating over energy and momentum, we take the limit $\delta \rightarrow 0$. This procedure is required in this singular perturbative calculation to fix the density of electrons $n=1$. Neglecting the momentum dependence of $\vec{s}_{\nu}(k)$ does not affect the leading singular behaviors. Then, we can carry out the calculation analytically. ${ }^{18}$ For $\varepsilon<0$, we obtain

$$
\operatorname{Re} \Sigma(\varepsilon) \sim \text { const }-c U^{2} \sqrt{|\varepsilon|}-c \frac{U^{3}}{2 \sqrt{|\varepsilon|}}+c \frac{U^{4}}{4|\varepsilon|^{3 / 2}}
$$

where $c$ is a positive constant of order unity. The real part of the self-energy for holelike excitations is divergent. The same divergent behavior appears in the imaginary part, however, for particlelike excitations. Higher-order corrections also show stronger divergence. This divergent behavior means that the unperturbed state is unstable in the presence of electron correlation and that the single-particle energy gap is generated. To see this, we take only up to the third-order term of the self-energy. Holelike excitations are possible only for the $E_{k 3}$ band in the half-filling case at zero temperature. Thus the single-particle energy of this band measured from the Fermi level is changed to $\varepsilon_{k} \sim-U^{2} \equiv-\Delta$ for $k$ $=0$. The $E_{k 3}$ band is pushed down to the lower energy, and the energy gap opens between the flat bands and $E_{k 3}$ band. This gap generation due to electron correlation signifies that the systems is in the Mott insulating state. Although the exact magnitude of the gap should be determined by taking into account higher-order corrections in $U$, the gapful state is the self-consistent solution for nonvanishing $U$. Thus an infinitesimally small $U$ drives the system into the Mott insulator at the half-filling. In the ground state, the two bands below the gap, $E_{k 3}, E_{k 4}$, are filled with up and down spins. Hence the system is in the spin singlet state. It should be stressed that, after the mass gap generation, the singularities of the perturbative expansion are eliminated, and as a result, the gap state is stabilized even if one takes into account higher-order corrections, which just renormalize the magnitude of the gap. 


\section{B. Quantum spin liquid state}

To see the magnetic property of the Mott insulating state obtained in the preceding section, we compute spin-spin correlation functions, $\chi_{\mu \nu}(q, \omega)=\left\langle S_{\mu}^{+}(q, \omega) S_{\nu}^{-}(-q,-\omega)\right\rangle$, where $S_{\nu}^{+}=\Sigma_{k} c_{k+q \nu \uparrow}^{\dagger} c_{k \nu \downarrow}$. We carry out perturbative expansion in terms of $U$ using the Green's function of the gap state, $G^{(33)}(\varepsilon) \sim\left[\varepsilon-E_{k 3}+\Delta\right]^{-1}$, as the unperturbed propagator. Calculating the diagrams up to the third order in $U$, we have $\operatorname{Im} \chi(q, \omega) \sim \sqrt{\omega-\Delta} \Theta(\omega-\Delta)$ with $\Theta(x)$ a step function. The spin gap exists between the spin singlet ground state and the triplet state. Thus the system is in the quantum disordered spin liquid state without magnetic long-range order. The result may not be changed qualitatively by higherorder corrections. These results are consistent with the previous studies for the pyrochlore Heisenberg model. ${ }^{8,12-15}$ The singularity of the self-energy (7) is deeply related with the accidental degeneracy at the $\Gamma$ point in the momentum space, which may cause an instability of lattice structure. This property may be relevant to the metal-insulator transition of $\mathrm{Tl}_{2} \mathrm{Ru}_{2} \mathrm{O}_{7}$, that accompanies a structure change. ${ }^{3,19}$

\section{THE HOLE-DOPED CASE}

\section{A. Heavy-fermion state}

In the hole-doped case, the singularities appeared in the half-filling case are eliminated by the presence of the cutoff which is the chemical potential $\mu$ measured from the halffilling level. Then the Fermi-liquid metallic state is realized. However, in the vicinity of the half-filling, the effective mass is much enhanced by electron correlation. Up to the third order in $U$, the leading term of the mass enhancement factor $z_{k}$ is

$$
z_{k}^{-1} \equiv 1-\left.\frac{\partial \operatorname{Re} \Sigma(\varepsilon)}{\partial \varepsilon}\right|_{\varepsilon \rightarrow 0} \sim \frac{U^{2}}{\sqrt{|\mu|}}+\frac{U^{3}}{|\mu|^{3 / 2}} .
$$

As the electron filling approaches the half-filling value $|\mu|$ $\rightarrow 0$, the mass enhancement factor shows the divergent behavior indicating the precursor of the Mott transition. Such a large mass enhancement is actually observed in the specific heat measurement for some pyrochlore itinerant systems such as $\mathrm{Y}(\mathrm{Sc}) \mathrm{Mn}_{2}$.

\section{B. Magnetic properties and the neutron cross section}

The largely enhanced effective mass obtained in the preceding section is a remarkable property of geometrically frustrated electron systems in which several modes of spin fluctuations compete with each other, and magnetic longrange order is suppressed. The spin fluctuation is almost localized in real space as in the case of $f$-electron-based heavyfermion systems. This property is seen more clearly in the momentum dependence of the spin-spin correlation function. We calculate it by the perturbative calculation. Since the magnetic frustration is so strong, any approximations that neglect the coupling between several modes of spin fluctuation such as random phase approximation (RPA) or fluctuation exchange approximation (FLEX) are invalid for this system. Actually the most singular contributions up to the order
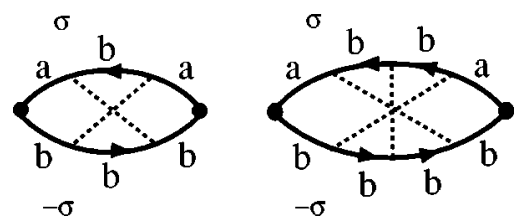

FIG. 3. The most singular diagrams of spin-spin correlation functions up to the third order in $U$.

of $O\left(U^{3}\right)$ comes from the diagrams shown in Fig. 3. To simplify the calculation we exploit the following approximation. Near the half-filling, the momenta running in the $G^{(33)}$ line take small values in the vicinity of the Fermi surface. Moreover, for small $k, \vec{s}_{3}(k)$ does not depend on $|k|$. Thus we can separate the integral over $|k|$ and the angle for the momenta running in $G^{(33)}$ line. Then, the spin-spin correlation function is decomposed into the momentum part and the energy part, $\chi_{\mu \nu}(q, \omega)=f(\omega) g_{\mu \nu}(q)$. Such a factorization of the spin-spin correlation function is indeed observed in an inelastic neutron scattering experiment for $\mathrm{Y}(\mathrm{Sc}) \mathrm{Mn}_{2} .{ }^{16} \mathrm{The}$ energy part is given by

$$
f(\omega) \sim-a_{1} U^{2} \ln \frac{|\omega+2 \mu|}{E_{c}}-a_{2} U^{3} \frac{1}{\omega+2 \mu}+\cdots
$$

for small $\omega$. Here $a_{1}$ and $a_{2}$ are constants. Thus in the vicinity of the half-filling, spin fluctuation is much enhanced. The presence of the giant spin fluctuation is also consistent with several experiments for itinerant pyrochlore systems. ${ }^{1,16}$ It is noted that such a large enhancement of $\chi_{\mu \nu}(q, \omega)$ is not obtained by RPA or FLEX approximations for the appropriate value of $U$. We calculate the structure factor $g_{\mu \nu}(q)$ numerically. In Fig. 4(a), we plot the intensity of $g_{12}(q)$ in the [110]-[001] plane of the reciprocal lattice. $g_{12}(q)$ is almost constant in the [001] direction, indicating that the spin fluctuation is strongly localized in this direction. It also has a small peak on the $q_{x}=q_{y}=0$ line showing the presence of small fluctuation toward a collinear magnetic order, as was pointed out in the study of the Heisenberg model. ${ }^{8}$ However this mode competes with the other modes that exist in $g_{13}(q), g_{23}(q), g_{14}(q), g_{24}(q)$, and thus such a magnetic order is suppressed.

In Fig. 4(b), we show the calculated result of the neutron cross section, $\sum_{\mu, \nu} e^{-i G\left(R_{\mu}-R_{\nu}\right)} \chi_{\mu \nu}(q, \omega)$, in the [110]-[001] plane. Here $G$ is a reciprocal lattice vector. The positions of the maximum are almost consistent with the neutron scattering experiment for $\mathrm{Y}(\mathrm{Sc}) \mathrm{Mn}_{2} \cdot{ }^{16}$ Note that this structure is very similar to that found for the Heisenberg model. ${ }^{8}$ Thus the itineracy of electron affects little the structure in the momentum space, though the dynamical properties between the Mott insulating state and the metallic state are quite different; namely, the former has gaps and the latter is gapless. The momentum dependence of $\chi_{\mu \nu}(q, \omega)$ implies that the spin fluctuation is localized in a tetrahedron forming collective four-spin singlet. ${ }^{10,8}$ Such a localized character of spin fluctuation is very similar to magnetic properties of $f$-electron-based heavy-fermion systems. However, in the latter systems, magnetic long-range order is suppressed by the 
(a)

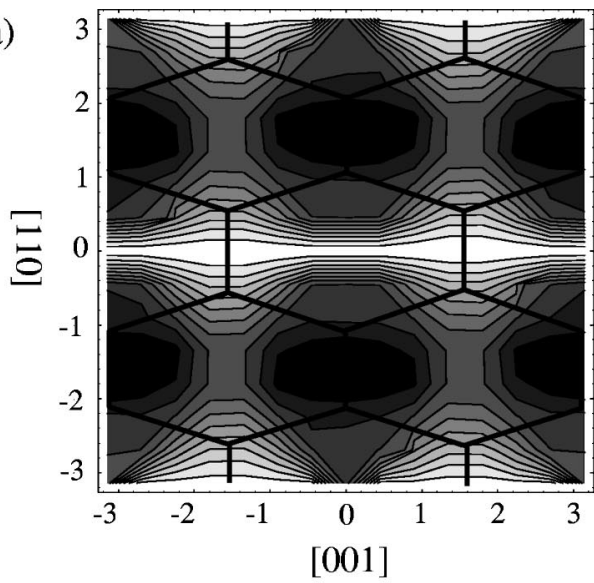

(b)

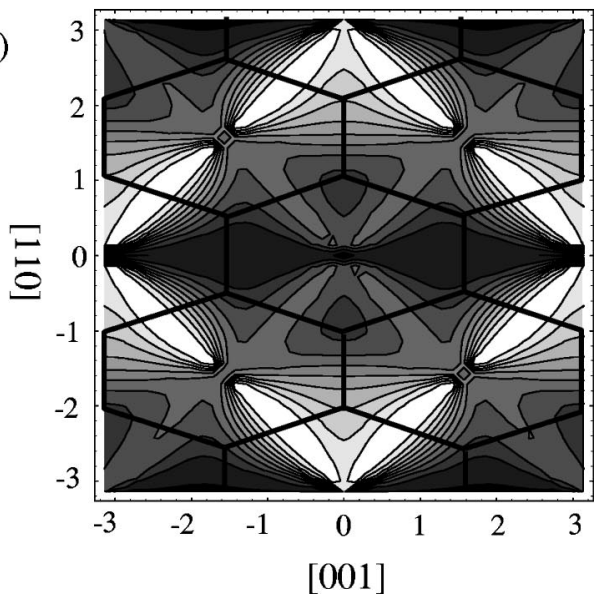

FIG. 4. (a)The structure factor $g_{12}(q)$ plotted in the [001]-[110] plane. The black line denotes the zone boundary. The brighter regions have the stronger intensity. (b) The neutron cross section.

presence of Kondo temperature higher than magnetic ordering temperature. In our system, the geometrical frustration is crucial.

\section{Compressibility}

Here we discuss the charge response of the heavy-fermion state near half-filling. In general, the charge susceptibility $\chi_{c}$ in the vicinity of the Mott metal-insulator transition point shows quite different behaviors depending on the density of states near the Fermi level. ${ }^{20}$ In our systems, the bare density of states in the hole-doped case is nonsingular, though fourpoint vertices are much enhanced by electron correlation. The perturbative calculation of the charge susceptibility gives singular terms in the limit of $\mu \rightarrow 0$, namely, $\chi_{c}$ $\sim C_{1} U^{2} \ln |\mu|+C_{2} U^{3} /|\mu|+\cdots$. Thus it is highly nontrivial how the charge susceptibility behaves, as the electron filling approaches the half-filling value. To see this we reiterate the most singular terms of the perturbative expansion in $U$. As was done before, we neglect the momentum dependence of $\vec{s}_{\nu}$. We first consider the terms that contain only one irreducible four-point vertex. The most singular terms of this type are diagrammatically expressed as Fig. 5(a). These terms are calculated as (a)
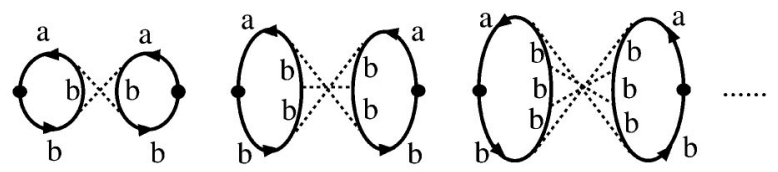

(b)

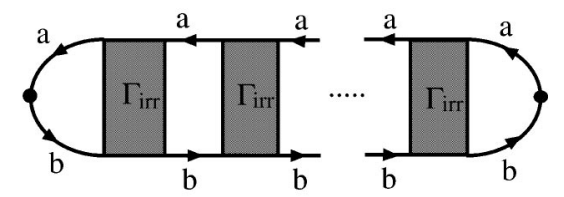

FIG. 5. Diagrams of the charge susceptibility. $\Gamma_{\text {irr }}$ is the irreducible 4-point vertex.

$$
\chi_{c}^{\mathrm{irr}}=C_{2} U^{2} \ln |\mu|+C_{3} U^{3} /|\mu|+C_{4} U^{4} /|\mu|^{2}+\cdots .
$$

Here $C_{2}, C_{3}$, and $C_{4}$ are positive constants. On the other hand, the term that contains $n$ irreducible four-point vertices shown in Fig. 5(b) gives the contribution $\sim\left[-c \chi_{c}^{\mathrm{irr}}\right]^{n} /|\mu|^{(n-1) / 2}$ with $c$ a positive constant. Summing up all most singular terms, we have,

$$
\chi_{c}=a \sqrt{|\mu|}-\frac{\chi_{c}^{\mathrm{irr}}}{1+(c / \sqrt{|\mu|}) \chi_{c}^{\mathrm{irr}}} .
$$

Thus as the electron filling approaches the half-filling value $\mu \rightarrow 0$, the charge susceptibility decreases toward zero, $\chi_{c}$ $\rightarrow 0$, indicating that the system becomes incompressible.

\section{SUMMARY}

In this paper, we investigate the Mott transition, strong correlation effects and spin dynamics of the pyrochlore Hubbard model. We show that, at the half-filling, electronelectron interaction leads the system into the Mott insulator in which the quantum disordered spin liquid state realizes. In the hole-doped case, we show that the effective mass is anomalously enhanced in the vicinity of the half-filling signifying the heavy-fermion state. We have also calculated the neutron cross section of this state, which is consistent with the recent experiment for some itinerant pyrochlore systems like $\mathrm{Y}(\mathrm{Sc}) \mathrm{Mn}_{2}$.

Although we carried out the perturbative calculation up to the fourth order in $U$, the singular divergent behavior of the self-energy shown in Sec. III implies that we need to count all order singular diagrams. Since the most singular diagrams have the specific structure as discussed in Sec. III, we can carry out this program in principle. We would like to address this issue in the near future.

\section{ACKNOWLEDGMENTS}

The author would like to thank K. Yamada, K. Ueda, N. Kawakami, H. Tsunetsugu, and H. Ikeda for invaluable discussions. This work was partly supported by a Grant-in-Aid from the Ministry of Education, Science, and Culture, Japan. 
${ }^{1}$ M. Shiga, H. Wada, Y. Nakamura, J. Deportes, and K.R.A. Ziebeck, J. Phys. Soc. Jpn. 57, 3141 (1988); M. Shiga, K. Fujisawa, and H. Wada, ibid. 62, 1329 (1993).

${ }^{2}$ S. Kondo, D.C. Johnston, C.A. Swenson, F. Borsa, A.V. Mahajan, L.L. Miller, T. Gu, A.I. Goldman, M.B. Maple, D.A. Gajewski, E.J. Freeman, N.R. Dilley, R.P. Dickey, J. Merrin, K. Kojima, G.M. Luke, Y.J. Uemura, O. Chmaissen, and J.D. Jorgensen, Phys. Rev. Lett. 78, 3729 (1997).

${ }^{3}$ T. Takeda, M. Nagata, H. Kobayashi, R. Kanno, Y. Kawamoto, M. Takano, T. Kamiyama, F. Izumi, and A.W. Sleight, J. Solid State Chem. 140, 182 (1998).

${ }^{4}$ Y. Taguchi and Y. Tokura, Phys. Rev. B 60, 10280 (1999).

${ }^{5}$ S. Yoshii and M. Sato, J. Phys. Soc. Jpn. 68, 3034 (1999).

${ }^{6}$ D. Mandrus, J. R. Thompson, R. Gaal, L. Forro, J. C. Bryan, B. C. Chakoumakos, L. M. Woods, B. C. Sales, R. S. Fishman, and N. Keppens, Phys. Rev. B 63, 195104 (2001).

${ }^{7}$ R. Moessner and J.T. Chalker, Phys. Rev. Lett. 80, 2929 (1998).

${ }^{8}$ B. Canals and C. Lacroix, Phys. Rev. Lett. 80, 2933 (1998); Phys. Rev. B 61, 1149 (2000).
${ }^{9}$ E.H. Lieb and P. Schupp, Phys. Rev. Lett. 83, 5362 (1999).

${ }^{10}$ M. Isoda and S. Mori, J. Phys. Soc. Jpn. 67, 4022 (1998).

${ }^{11}$ Y. Yamashita and K. Ueda, Phys. Rev. Lett. 85, 4960 (2000).

${ }^{12}$ M.J.P. Gingras, B.C. den Hertog, M. Faucher, J.S. Gardner, S.R. Dunsiger, L.J. Chang, B.D. Gaulin, N.P. Raju, and J.E. Greedan, Phys. Rev. B 62, 6496 (2000).

${ }^{13}$ A. Koga and N. Kawakami, Phys. Rev. B 63, 0144432 (2001).

${ }^{14}$ H. Tsunetsugu, J. Phys. Soc. Jpn. 70, 640 (2001).

${ }^{15}$ B. Canals and D. Garanin, cond-mat/0102237 (unpublished).

${ }^{16}$ R. Ballou, E. Lelièvre-Berna, and B. Fak, Phys. Rev. Lett. 76, 2125 (1996)

${ }^{17}$ M. Isoda and S. Mori, J. Phys. Soc. Jpn. 69, 1509 (2000).

${ }^{18}$ See, for example, A. A. Abrikosov, L. P. Gorkov, and I. E. Dzyaloshinskii, Quantum Field Theoretical Methods in Statistical Physics (Pergamon Press, New York, 1965).

${ }^{19}$ F. Ishii and T. Oguchi, J. Phys. Soc. Jpn. 69, 526 (2000).

${ }^{20}$ M. Imada, A. Fujimori, and Y. Tokura, Rev. Mod. Phys. 70, 1039 (1998). 